This item was submitted to Loughborough's Research Repository by the author.

Items in Figshare are protected by copyright, with all rights reserved, unless otherwise indicated.

\title{
Solutions of the hyperbolic sine-Gordon equations
}

PLEASE CITE THE PUBLISHED VERSION

LICENCE

CC BY-NC-ND 4.0

\section{REPOSITORY RECORD}

Hoenselaers, C.. 2019. "Solutions of the Hyperbolic Sine-gordon Equations". figshare. https://hdl.handle.net/2134/578. 


\title{
Solutions of the Hyperbolic sine-Gordon Equations
}

\author{
C. Hoenselaers \\ Department of Mathematical Sciences \\ Loughborough University \\ Loughborough, LE11 3HE, UK
}

Nov. 2005

\section{Abstract}

We study soliton solutions of the Sinh-Gordon equation. It is also shown that the Cosh-Gordon equation, whilst being integrable, does not admit pure solitons. 


\section{1) Introduction}

The sine-Gordon equation

$$
\left(\partial_{\mathbf{x}}^{2}-\partial_{\mathbf{t}}^{2}\right) \varphi=\partial_{\mathbf{u v}} \varphi=\sin \varphi
$$

(in space-time or double null coordinates) has been studied in many publications too numerous to list here, as one instance cf. [1]. Also, replacing the sine-function by the cosine-function does not yield anything new.

On the other hand, replacing the sine-function with its hyperbolic counterpart might lead to some new insights. In this short note we shall examine the hyperbolic counterparts - there are two of them, since there is no real transformation between the Sinh and the Cosh - of the sine-Gordon equation.

\section{2) General Remarks}

We study the equations

$$
\begin{aligned}
& \partial_{\mathbf{u v}} \varphi=\operatorname{Sinh} \varphi \\
& \partial_{\mathbf{u v}} \varphi=\operatorname{Cosh} \varphi
\end{aligned}
$$

Both equations are known to be integrable and admit a pseudopotential, viz.

$$
\begin{aligned}
& \partial_{\mathbf{u}} \gamma=\partial_{\mathbf{u}} \varphi-\frac{1}{\lambda} \operatorname{Sinh} \gamma \\
& \partial_{\mathbf{v}} \gamma=\lambda \operatorname{Sinh}(\varphi-\gamma) \\
& \partial_{\mathbf{u}} \gamma=\partial_{\mathbf{u}} \varphi+\frac{1}{\lambda} \operatorname{Sinh} \gamma \\
& \partial_{\mathbf{v}} \gamma=\lambda \operatorname{Cosh}(\varphi-\gamma)
\end{aligned}
$$

The integrability conditions for (2a) and (2b) yield the equations (1a) and (1b). With the help of the pseudopotential a new solution is given in both cases via a Bäcklund transformation as

$$
\widetilde{\varphi}=\varphi-2 \gamma
$$

Each Bäcklund transformation generates a soliton. The difference between the two equations is that for the Sinh-Gordon equation one has a trivial solution, $\varphi=0$, to start the process whereas for the Cosh-Gordon equation this solution does not exist. This leads to the suspicion that the Cosh-Gordon equation, whilst being integrable, does not admit pure solitons.

Since there appears to be no generally accepted definition of a "soliton", we shall denote, in the present context, a localized "lump" of energy, to be described by the Hamiltonian of the system in question. 


\section{3) Some Solutions}

We shall first present solutions for the Sinh-Gordon equation in space-time coordinates, viz.

$$
\partial_{\mathbf{x}}^{2} \varphi-\partial_{\mathbf{t}}^{2} \varphi=\operatorname{Sinh} \varphi
$$

We take this solution to be a travelling wave which, by the Lorentz invariance of the equation, can be taken to be in its rest frame, hence $\varphi=\varphi(x)$. Multiplying (4) by $\partial_{x} \varphi$ and integrating we get

$$
\frac{1}{2} \partial_{\mathrm{X}} \varphi^{2}=\operatorname{Cosh} \varphi-\mathrm{E}
$$

the solution of which is given in terms of Jacobi elliptic functions (in the notation we follow [2]) as

$$
\varphi=2 \ln \left(\sqrt{\mathrm{k}} \operatorname{sn}\left(\frac{\mathrm{x}}{2 \sqrt{\mathrm{k}}}, \mathrm{k}\right)\right), \quad \mathrm{E}=\frac{1}{2}\left(\mathrm{k}+\frac{1}{\mathrm{k}}\right)
$$

In particular, for $\mathrm{k}=1, \mathrm{E}=1$, this solution degenerates into the 1 -soliton solution which can be rewritten as

$$
\varphi=4 \operatorname{ArTanh} \mathrm{e}^{-2 \mathrm{x}}
$$

In analogy to the procedure for the sine-Gordon equation the 2-soliton solution in its rest frame is obtained from the Ansatz [3]

$$
\varphi=4 \operatorname{Ar} \operatorname{Tanh}(\mathrm{a}(\mathrm{x}) \mathrm{b}(\mathrm{t}))
$$

$a$ and $b$ have to satisfy

$$
\begin{gathered}
\partial_{x} a^{2}=c_{0}+c x^{2}+c_{4} x^{4} \\
\partial_{t} b^{2}=c_{4}+(c-1) x^{2}+c_{0} x^{4}
\end{gathered}
$$

The 2-soliton solution is given by either $c_{O}=0$ or $c_{4}=0$. A typical solution, for $\mathrm{c}_{\mathrm{O}}=0$, reads

$$
\begin{aligned}
& a=\sqrt{\frac{c}{c_{4}}} \frac{1}{\operatorname{Sinh}(\sqrt{c} x)} \\
& b=\sqrt{\frac{c 4}{c-1}} \operatorname{Sinh}(\sqrt{c-1} t)
\end{aligned}
$$

Generally, the behaviour of Sinh-Gordon solitons is rather similar to that of sineGordon solitons with the exception that Sinh-Gordon solitons are given by singularities rather than wave crests. The Lagrangian is given by

$$
L=\frac{1}{2}\left(\partial_{x} \varphi^{2}-\partial_{t} \varphi^{2}\right)+\operatorname{Cosh} \varphi
$$


and the Hamiltonian, the value of which, for a given solution, is the energy density, reads (the additive constant stems from the convention to have the lowest state at zero energy.)

$$
H=\frac{1}{2}\left(\partial_{x} \varphi^{2}+\partial_{t} \varphi^{2}\right)+\operatorname{Cosh} \varphi-1
$$

For the 1-soliton solution the energy density is

$$
E=8 e^{2 x}\left(1-e^{2 x}\right)^{-2}
$$

While the integral over this energy density does not exist, its Cauchy principal value does exist and

$$
f_{-\infty}^{+\infty} \mathrm{E} d x=4
$$

We now turn to solutions of the Cosh-Gordon equation. In this case the equation reads

$$
\partial_{\mathrm{x}}^{2} \varphi-\partial_{\mathrm{t}}^{2} \varphi=\operatorname{Cosh} \varphi
$$

and, looking for a travelling wave solution in its rest frame, the analogue of (7) becomes

$$
\frac{1}{2} \partial_{x} \varphi^{2}=\operatorname{Sinh} \varphi-E
$$

This right-hand side of this equation does not admit a double zero and thus there are no solutions in terms of elementary functions. A solution is given by

$$
\varphi=-2 \ln \left(\frac{1}{\sqrt{k}} \operatorname{cn}\left(\frac{x}{2 \sqrt{k}}, k\right)\right), \quad E=\frac{1}{2}\left(k+\frac{1}{k}\right)
$$

The Hamiltonian for the Cosh-Gordon equation, viz.

$$
H=\frac{1}{2}\left(\partial_{X} \varphi^{2}+\partial_{t} \varphi^{2}\right)+\operatorname{Sinh} \varphi
$$

is not bounded from below and thus there is no ground state.

\section{4) Concluding remarks}

We have shown that integrability of an equation is not necessarily related to the existence of solitons. This is, in particular, the case if the equation in question does not admit a trivial solution and the Hamiltonian does not have a ground state. 


\section{References}

1) C. Rogers and W. K. Schief: Bäcklund and Darboux Transformations Cambridge University Press, Cambridge (2002)

R. Meinel, G. Neugebauer and H. Steudel: Solitonen Akademie Verlag, Berlin (1991)

2) I. S. Gradshteyn and I. M. Ryzhik: Table of Integrals, Series and Products Academic Press, New York (1965)

3) C. Hoenselaers and S. Miccichè:

Transcendental Solutions of the sine-Gordon Equation

Centre de Recherches Mathematiques, CRM Proceedings and Lecture Notes, vol. 29 (2001) 